\title{
Diabetes As an Independent Risk Factor for Stroke Recurrence in Ischemic Stroke Patients: An Updated Meta-Analysis
}

\author{
Li Zhang $^{\text {a }}$ Xianqi Li ${ }^{a}$ Charles D.A. Wolfe ${ }^{a, b, c}$ Matthew D.L. O'Connell ${ }^{a}$ \\ Yanzhong Wang ${ }^{a}, b, c$
}

aSchool of Population Health and Environmental Sciences, King's College London, London, UK; bNational Institute for Health Research (NIHR) Biomedical Research Centre (BRC), Guy's and St Thomas' NHS Foundation Trust and King's College London, London, UK; 'NIHR Applied Research Collaboration (ARC) South London, London, UK

\section{Keywords}

Diabetes · Ischemic stroke $\cdot$ Lacunar stroke $\cdot$ Stroke recurrence

\begin{abstract}
Introduction: Stroke and its recurrence and diabetes will increase in incidence as the population ages globally. This study explores the relationship between diabetes and stroke recurrence to understand if diabetes is an independent predictor for stroke recurrence in ischemic stroke (IS) patients. Methods: We conducted a systematic review and meta-analysis of studies on the effect of diabetes on stroke recurrence among patients with IS. We searched population-based studies published before 15th February 2021 in PubMed and EMBASE following PRISMA guidelines. Random-effects estimates of the pooled hazard ratio (HR) and $95 \%$ confidence intervals (Cls) of each study were generated. A funnel plot and an Egger test were performed to evaluate publication bias. All statistical analyses were conducted in the R software 4.0.1 and Stata 16.0. Results: The search identified 3,121 citations, of which 27 studies met inclusion criteria. Diabetes was associated with a significant risk of stroke recurrence in all IS patients (pooled HR, 1.50; 95\% Cl: $1.36-1.65 ; l^{2}=61.0 \%$ ). Sim-
\end{abstract}

ilar results were found in lacunar stroke patients with diabetes (pooled HR, 1.65; 95\% Cl: 1.41-1.92; $R^{2}=22.0 \%$ ). Moreover, we found that the risk of recurrent IS among patients of IS with diabetes was higher than that in those without diabetes (pooled HR, 1.53; 95\% Cl: $1.30-1.81 ; R^{2}=74.0 \%$ ). Conclusion: Diabetes is an independent risk factor for stroke recurrence among patients with IS.

(C) 2021 The Author(s)

Published by S. Karger AG, Basel

\section{Introduction}

Stroke is the second leading cause of death and a significant cause of disability worldwide [1], and it is projected to remain so until at least 2030 [2]. Ischemic stroke (IS) is one of the most common subtypes of stroke. According to the Global Burden of Disease Study 2016, in 2016, there were 80.1 million stroke cases globally, among which $84.4 \%$ were ISs. Stroke survivors are at high risk of having another stroke in the first 30 days following the first-ever stroke [3], and recurrent strokes are more likely to be disabling or fatal than the first-ever stroke [4].

Matthew D.L. O'Connell and Yanzhong Wang are joint senior authors. karger@karger.com www.karger.com/ned

Karger $\stackrel{\text { ' }}{5}$

BOPEN ACCESS
(C) 2021 The Author(s)

Published by S. Karger AG, Basel

This is an Open Access article licensed under the Creative Commons Attribution-NonCommercial-4.0 International License (CC BY-NC) (http://www.karger.com/Services/OpenAccessLicense), applicable to the online version of the article only. Usage and distribution for commercial purposes requires written permission.
Correspondence to:

Li Zhang, li.1.zhang@kcl.ac.uk 
Diabetes is a highly prevalent and growing chronic disease that affected about 415 million people worldwide in 2015 and is expected to affect 642 million people by 2040 [5]. Moreover, diabetes mellitus is a well-recognized risk factor for neurovascular disease [6-8], including IS [9], and a significant proportion of stroke patients have comorbid diabetes [10].

Given the severe health and economic burden of stroke and its recurrence and diabetes on individuals and society levels as the population ages, it is imperative to focus on the effect of diabetes on stroke recurrence and improve outcomes of patients with stroke and diabetes. A 2015 meta-analysis of 18 studies explored the association between diabetes and stroke recurrence in stroke patients. They found a hazard ratio (HR) of 1.44 (95\% confidence interval [CI]: 1.28-1.61) for the effect of diabetes on stroke recurrence after IS by a subgroup analysis of 14 studies [11]. However, there have since been a number of relevant studies published. This earlier meta-analysis was limited by the inclusion of heterogeneous samples of patients with all stroke subtypes and those with specific stroke-related syndromes. For example, the patients of one of their included studies were presented with IS with depression, executive dysfunction, and depression-executive dysfunction syndrome [12], which considered a population of IS with comorbid conditions identified from 1993 to 1995. Moreover, this meta-analysis included patients with transient ischemic attack (TIA), also called a mini-stroke. However, TIA has markedly different pathological features compared to stroke. To ensure the comparability between studies, we only consider patients with IS. Therefore, recent data from population-based studies on the relationship between diabetes and stroke recurrence among patients with IS are needed. The results will enable a better understanding of the etiology of stroke recurrence.

\section{Methods}

Search Strategy and Selection Criteria

A comprehensive search of PubMed and EMBASE was conducted to identify relevant studies published before 15th February 2021 with the search terms: diabetes AND stroke AND recurrence. A detailed search strategy is provided in the online supplementary Appendix 1; for all online supplementary material, see www.karger.com/doi/10.1159/000519327. This study followed the PRISMA reporting guideline for study selection, data collection and synthesis, assessment of bias, and quality assessment. To minimize reporting bias, this systematic review and meta-analysis were registered on PROSPERO (CRD42021248845).
Studies were included if (1) participants as patients with IS and its subtypes and subtype classification of IS was carried out using the Trial of ORG 10172 in Acute Stroke Treatment criteria-into large-artery atherosclerosis, small-vessel occlusion (or small-artery occlusion), cardioembolism, other determining etiologies, and undetermined etiologies [13]. Besides, patients with lacunar stroke or lacunar infarcts were also identified according to the criteria established by the Oxfordshire Community Stroke Project [14]; (2) they could be defined as a cohort study or a randomized clinical trial (RCT), either prospective or retrospective; (3) the outcome of interest was the first stroke recurrence, regardless of stroke subtypes during the follow-up period; and (4) multivariable-adjusted HR was reported with the $95 \%$ CIs. Studies were excluded if one of the following criteria existed: (1) other publication types (i.e., conference abstracts, case reports, letters, or reviews); (2) studies that included fewer than 10 participants; and (3) HR without $95 \%$ CIs or they could not be calculated through the reported data.

\section{Data Extraction}

After the databases searches, studies were identified with duplicates removed. One researcher (Li Zhang) conducted an initial screening of all titles and abstracts; Subsequently, 2 independent researchers (Li Zhang and Xianqi Li) evaluated all potentially relevant articles based on the inclusion criteria described above and jointly selected studies eligible for our systematic review. Disagreements between the 2 reviewers were resolved by consensus with the other 2 researchers in our team (Yanzhong Wang and Matthew O'Connell).

Data were independently extracted by 2 investigators (Li Zhang and Xianqi Li) and checked by the other authors. The following data were extracted into standardized forms: (1) study characteristics (authors, year of publication, study design, country, and sample size); (2) demographic and clinical characteristics (stroke types, age, and follow-up duration); and (3) outcome (stroke types and HR with 95\% CIs).

\section{Assessment of Study Quality}

Two researchers (Li Zhang and Xianqi Li) independently rated the quality of each article by using the Newcastle Ottawa Scale (NOS). The NOS was a 9-point scoring system developed for assessing the quality of cohort studies from 3 aspects: selection, comparability, and outcome. A study can be awarded a maximum of one star for each numbered item within the selection (maximum, 4 points) and outcome (maximum, 3 points). A maximum of 2 stars can be given for comparability. More details of NOS can be seen in the online supplementary Appendix 2. Studies that received an overall score of 7 or higher were considered good quality, 5-6 points were fair quality, and below 5 points were poor quality [15]. Any discrepancy was resolved by consensus.

\section{Statistical Analysis}

Effect measures of interest were multivariable-adjusted HR and the corresponding 95\% CIs. The pooled HR was obtained by a random-effects model with an inverse-variance weighting model. Statistical heterogeneity among studies was assessed using the Higgins inconsistency index $\left(I^{2}\right)$ test [16], and $I^{2}$ values of $25 \%, 50 \%$, and $75 \%$ were regarded as low, moderate, and high heterogeneity, respectively [17]. Publication bias was evaluated by a funnel plot and an Egger test [18], and $p<0.10$ values from an Egger test in- 
Fig. 1. PRISMA flowchart. OR, odds ratio; $\mathrm{RR}$, relative ratio; IS, ischemic stroke; CI, confidence interval; HR, hazard ratio; TIA, transient ischemic attack.

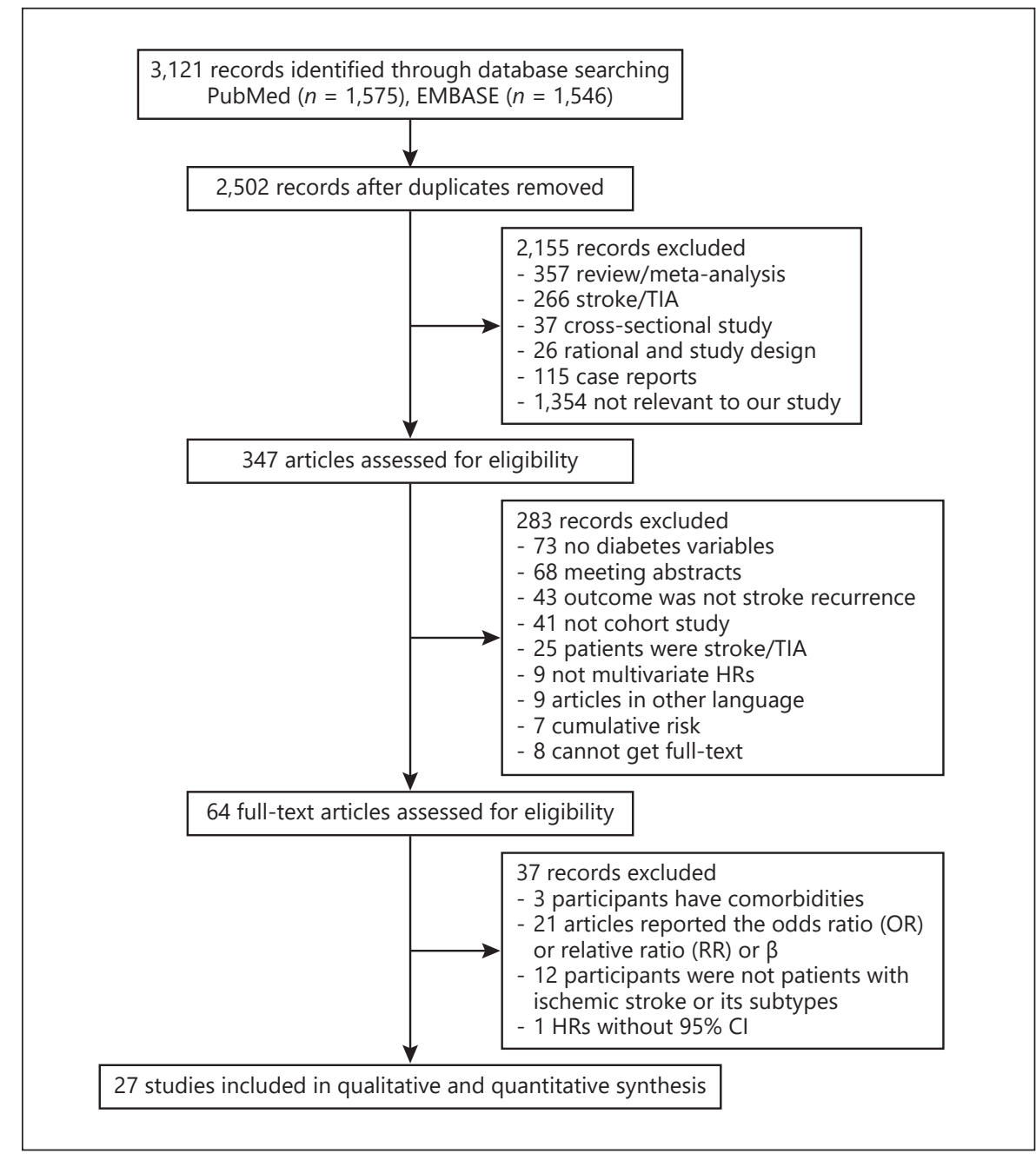

dicated statistically significant publication bias. All the above analyses were performed with the statistical software $\mathrm{R}$ version 4.0.1, using the package meta. Funnel plots were drawn with Stata 16.0. A $p$ value $<0.05$ was considered statistically significant. All statistical tests were two-sided.

\section{Results}

\section{Selection Process and Characteristics of Included Studies}

The search identified 3,121 possibly eligible articles from PubMed $(n=1,575)$ and EMBASE $(n=1,546)$. We screened 2,502 articles by titles and abstracts after duplicates were removed. Based on further assessment, 2,438 articles were excluded. Sixty-four articles were relevant for full-text review, and 27 met the inclusion criteria and were finally enrolled in this meta-analysis (Fig. 1). Table 1 describes the characteristics of the 27 included studies.
All these articles were published after 1990, in which the study period ranged from 1983 to 2018 . The sample size for each study varied from 100 to 196,765 , with a total of 274,631 patients. Twenty-four studies were retrospective or prospective cohort studies, and 3 studies were retrospective analyses of RCTs. Of the 27 studies, the participants of 20 studies were patients with IS without specific subtypes, 6 studies were lacunar stroke (or small-artery occlusion), and one study was cardio-embolism stroke. The outcome of 11 studies was recurrent ISs, and in the remaining 16 studies, their outcome was recurrent strokes without specific subtypes. The average length of the follow-up for each study ranged from 3 months to 5 years. The 27 included studies were from China $(n=8)$, United States $(n=5)$, Japan $(n=5)$, Sweden $(n=2)$, Korea $(n=$ $1)$, Greece $(n=1)$, Finland $(n=1)$, and Australia $(n=1)$; 2 were from a worldwide RCT involving 81 centers; and one was from an RCT involving 35 countries. 
Table 1. Characteristics of the studies included in the meta-analysis [23-33, 45-60]

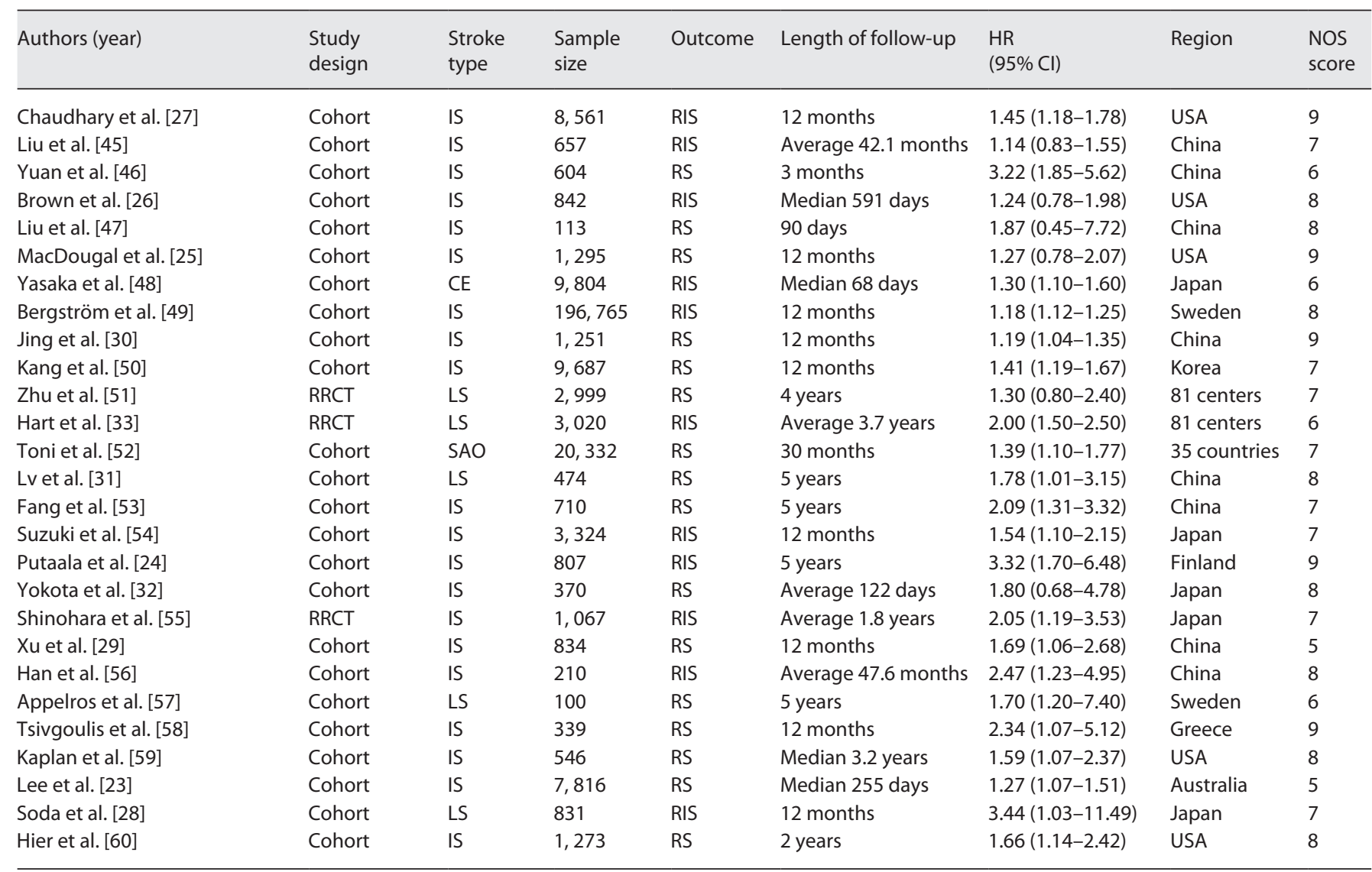

RRCT, retrospective analysis of randomized clinical trial; IS, ischemic stroke; LS, lacunar stroke; CE, cardio-embolism stroke; SAO, small-artery occlusion; $\mathrm{RS}$, recurrent stroke; RIS, recurrent ischemic stroke; HR, hazard ratio; $\mathrm{Cl}$, confidence interval.

The Quality Assessment of Included Studies

The NOS score allocated for each study ranged from 5 to 9 points, with a mean value of 7 points (online suppl. Table 1). There were in total 21 studies with NOS scores equal to or greater than 7 points, which indicated the quality of these studies was considered methodologically good, and the remaining 6 studies were regarded as fair quality.

\section{Association between Diabetes and Stroke Recurrence in IS Patients}

As shown in Figure 2, the pooled HR was 1.50 (95\% CI: 1.36-1.65), indicating that the risk of stroke recurrence in IS patients with diabetes was 1.50 times higher than in those without diabetes. Moderate heterogeneity was observed $\left(I^{2}=61.0 \% ; p<0.01\right)$. Visual inspection of the funnel plot (online suppl. Fig. 1) indicated asymmetry, and the Egger test suggested publication bias $(p<0.0001)$.

\section{Association between Diabetes and Recurrent IS in IS Patients}

As shown in Figure 3, the pooled HR was 1.53 (95\% CI: 1.30-1.81), indicating that the risk of recurrent IS in IS patients with diabetes was 1.53 times higher than in those without diabetes. Moderate heterogeneity was observed $\left(I^{2}=74.0 \% ; p<0.01\right)$. Visual inspection of the funnel plot (online suppl. Fig. 2) indicated asymmetry, and the Egger test suggested publication bias $(p<$ $0.0001)$.

\section{Association between Diabetes and Stroke Recurrence in Lacunar Stroke Patients}

As shown in Figure 4, the pooled HR was 1.65 (95\% CI: 1.41-1.92) through the fixed-effect model, indicating that the risk of stroke recurrence in lacunar stroke patients with diabetes was 1.65 times higher than in those without diabetes. Low heterogeneity was observed $\left(I^{2}=22.0 \%\right.$; 


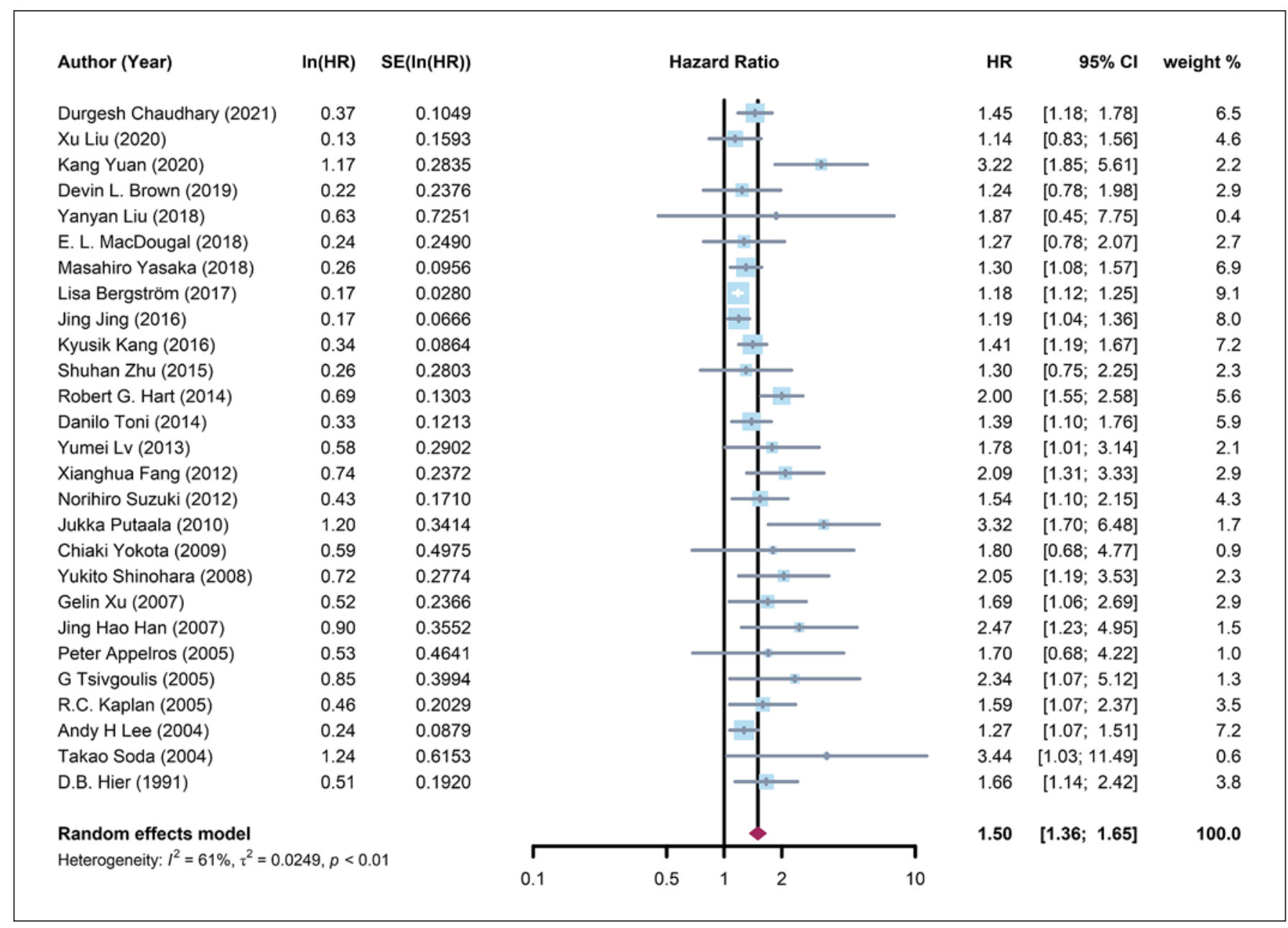

Fig. 2. Forest plot of the association of diabetes with risk of stroke recurrence in IS patients. IS, ischemic stroke; 95\% CI, 95\% confidence interval; lnHR, the natural logarithm of hazard ratio; selnHR, standard error of the $\ln H \mathrm{R}$; $\mathrm{HR}$, hazard ratio.

$p=0.27)$. It was impossible to conduct a publication bias test because of the small number of the included studies $(n=6)$ in this analysis.

\section{Discussion}

This systematic review and meta-analysis summarized the available data on the effects of diabetes on stroke recurrence among patients with IS and its subtypes. We found a significant stroke recurrence risk in IS patients with diabetes (pooled HR, 1.50; 95\% CI: 1.36-1.65; $I^{2}=$ $61.0 \%)$ compared to those without diabetes. Similar results were found in lacunar stroke patients with diabetes (pooled HR, 1.65; 95\% CI: 1.41-1.92; $I^{2}=22.0 \%$ ). More- over, the risk of recurrent IS among patients of IS with diabetes was higher than that in those without diabetes (pooled HR, 1.53; 95\% CI: 1.30-1.81; $I^{2}=74.0 \%$ ). These findings suggest that diabetes is an independent risk factor for stroke recurrence in IS patients.

Diabetes is a highly prevalent chronic disease and a well-recognized risk factor for incident IS [9]. However, results regarding its role on outcomes after stroke have been inconsistent due to differences in the study population, length of follow-up, selection of covariates, and diagnostic criteria for diabetes and stroke outcomes [1922]. Compared with the HR of 1.44 (95\% CI, 1.28-1.61) in the 2015 meta-analysis [11], we found an increased stroke recurrence risk of 1.50 (95\% CI, 1.36-1.65) for IS patients with diabetes, raising the need for management 


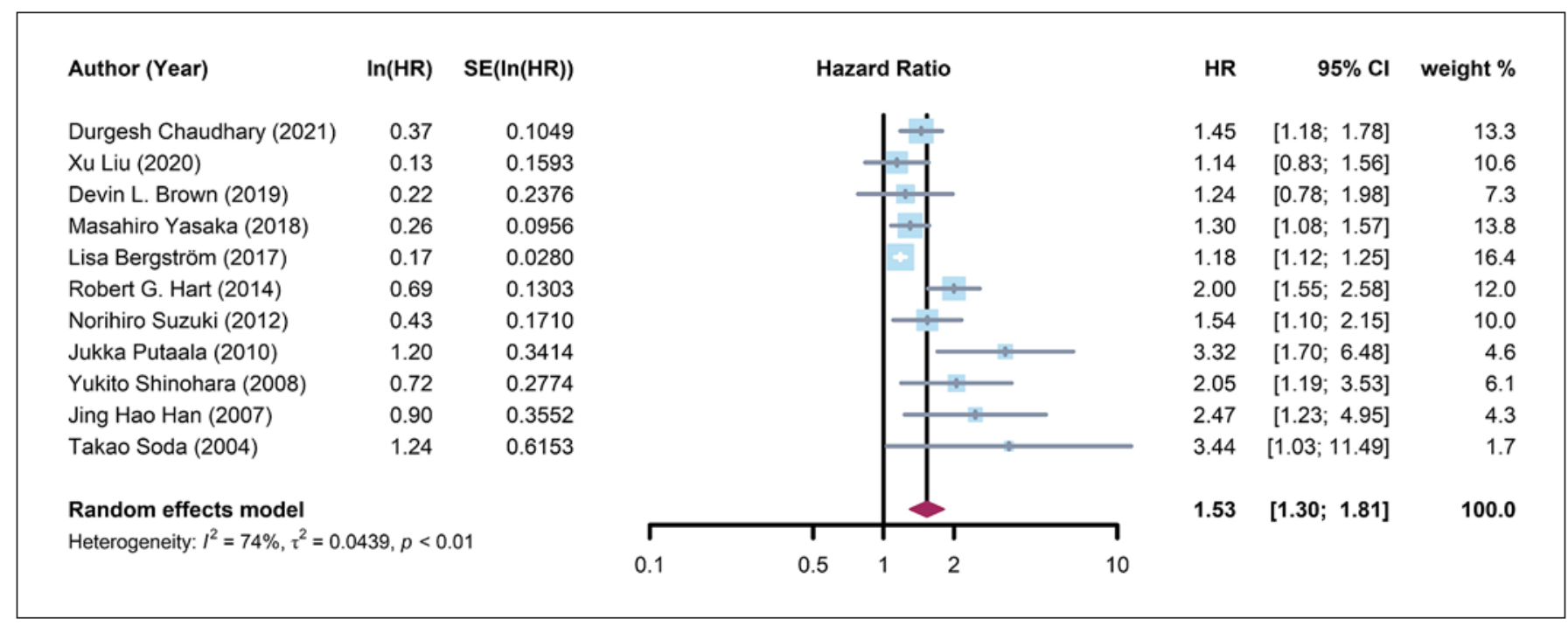

Fig. 3. Forest plot of the association of diabetes with risk of IS recurrence in IS patients. 95\% CI, 95\% confidence interval; lnHR, the natural logarithm of hazard ratio; selnHR, standard error of the lnHR; IS, ischemic stroke; HR, hazard ratio.

of diabetes in IS patients. Apart from the strong associations observed between diagnosed diabetes and vascular outcomes, a meta-analysis conducted in 2010 involving 102 cohorts in 25 countries showed a much more moderate relationship of impaired fasting glucose status with coronary heart disease and stroke [9]. Therefore, it is reasonable to think that impaired fasting glucose may also be associated with stroke recurrence. However, large-scale RCTs or epidemiological studies are still needed to confirm this association. In addition to diabetic populations, we should also include patients with prediabetes or impaired fasting glucose in the monitoring and management to avoid worse outcomes.

This study has several limitations. First, most of the included studies were retrospective analyses. Large-scale prospective studies are needed in the future. Second, we found low $\left(I^{2}=22.0 \%\right)$ to moderate heterogeneity $\left(I^{2}=\right.$ $\left.61.0 \%, I^{2}=74.0 \%\right)$ in the present study. Apart from differences in sample size, length of follow-up, and selection of covariates, an important observation is the difference in diagnosing diabetes. Most of these studies linked hospital records to ascertain the medical history of participants [23-27], while some diagnosed diabetes via a level of fasting glucose and HbA1c [28-30]. Self-reported diabetes and patients treated with antidiabetic medication or insulin were also the criteria [31-33]. These differences limited the comparability of results. Third, diabetes often co-occurs with other cardiovascular risk factors, particu- larly hypertension and hypercholesterolemia, which are also associated with a higher risk of recurrence. Therefore, it is not entirely clear whether the increased risk of recurrence in people with diabetes reflects a direct effect of their diabetes or the overall burden of morbidity. Although this should be partially accounted for by a multivariable adjustment in some studies, it was not possible to separate out a pure effect of diabetes from the published studies as no stratified data or analyses were presented. Fourth, publication bias was present in pooling the HR, probably owing to small-study effects [34]. Also, we could not exclude the risk of publication bias against studies reporting negative findings. Thus, the findings of the present meta-analysis should be read with caution during the interpretation of conclusions.

Despite these limitations, this meta-analysis provided a comprehensive overview of the role of diabetes in stroke recurrence in IS patients. Based on the previous meta-analysis conducted in 2015, we updated the results by searching databases comprehensively and finally included and synthesized a broad range of relevant articles. Compared with the 2015 meta-analysis, we have more restrictive inclusion and exclusion criteria to ensure the quality of the metaanalysis and interpretability of the results. First, we only focused on IS patients instead of all strokes. TIA, also called a mini-stroke, was not included in the current study due to its markedly different pathological features compared to stroke. Second, to ensure the comparability between arti- 


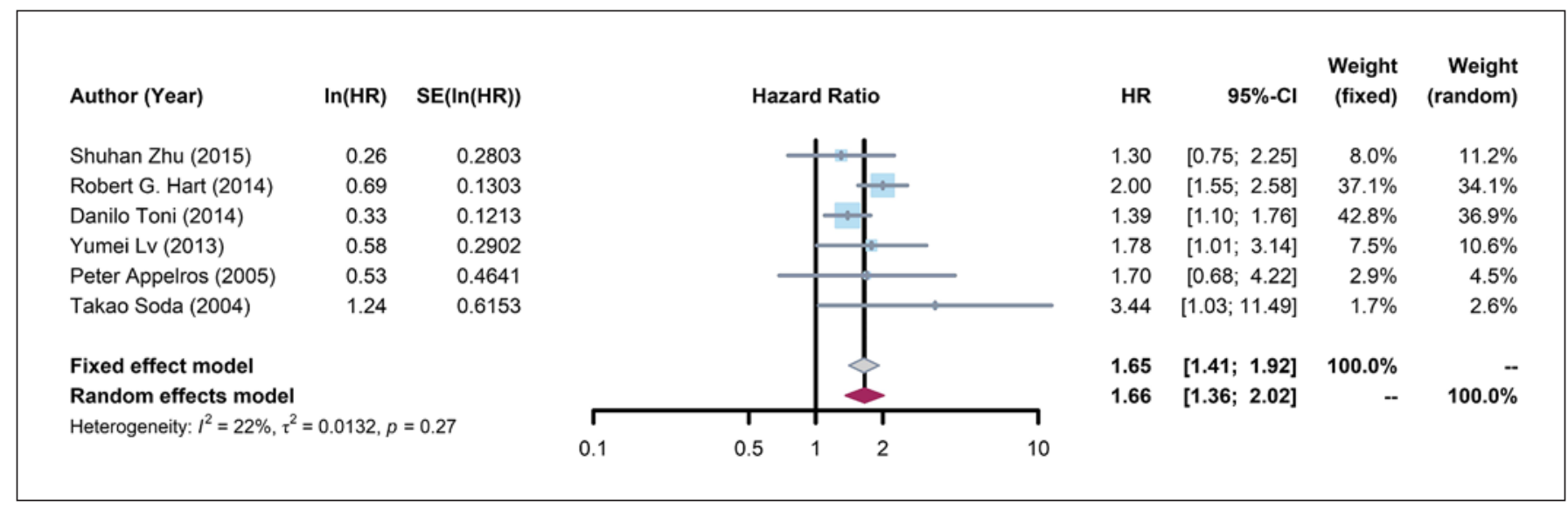

Fig. 4. Forest plot of the association of diabetes with risk of stroke recurrence in lacunar stroke patients. 95\% CI, 95\% confidence interval; lnHR, the natural logarithm of hazard ratio; selnHR, standard error of the lnHR; $\mathrm{HR}$, hazard ratio.

cles, we only considered participants with IS instead of IS patients who have comorbid conditions, for instance, IS patients with moyamoya disease [35] or IS with depression [12]. Moreover, despite the small number of included studies of lacunar stroke, a subgroup analysis was conducted. We found that diabetes played an essential role in the risk of stroke recurrence in lacunar stroke patients.

Overall, this meta-analysis highlighted the importance of detecting diabetes post-IS and recognizing the potential for stroke recurrence. Further research of this area is needed to specify the effect of diabetes on stroke recurrence. First, although type 2 diabetes is more prevalent and accounts for roughly $90 \%$ of all diabetes cases worldwide [36], we cannot ignore the prognosis of stroke patients with type 1 diabetes. Studies for the effect of specific diabetes subtypes on stroke recurrence will be more valuable. Second, despite that fasting blood glucose remains a commonly measured glycemic parameter to identify diabetic patients [37], its accuracy in acute stroke might be affected by stress hyperglycemia [38-40]. This well-established phenomenon occurs after any acute illness. For instance, stress hyperglycemia has been found to be associated with worse outcomes in acute hemorrhagic stroke patients [41-43]. This risk is likely to be underestimated, given that diabetic status was often recorded from medical records. Therefore, unrecognized diabetic patients were included in the nondiabetic group. Besides, HAlbc was one of the diagnosis criteria of diabetes, indicating the chronic glycemic status, which has been found to be associated with stroke recurrence after stroke [44]. However, this criterion has not been fully used in the projects conducted in recent years. Therefore, a combination of HAlbc with glucose testing and even more strict standards like the diagnosis of stress hyperglycemia should be warranted in future research projects to avoid underestimating the effect of diabetes on stroke recurrence. Third, the effect of diabetes alone, in the absence of hypertension and hypercholesterolemia, on stroke recurrence among patients with IS remains an important question to address in future studies. Fourth, only a few studies have assessed patients with hemorrhagic stroke, raising the importance of diabetes and its management in hemorrhagic stroke.

In conclusion, the present meta-analysis incorporated studies over the past 30 years, giving the latest overview and understanding of the relationship between diabetes and stroke recurrence in IS patients. Results showed that diabetes is an independent risk factor for stroke recurrence in IS patients, but more extensive prospective studies, as well as consistent definitions of diabetes and stroke recurrence measures, are needed in future to provide robust evidence to guide management.

\section{Acknowledgements}

The authors acknowledge support from the National Institute for Health Research (NIHR), Applied Research Collaboration (ARC), South London at King's College Hospital (KCH), NHS Foundation Trust, the NIHR Biomedical Research Centre (BRC), Guy's and St Thomas' NHS Foundation Trust and King's College London, UK, and the program of the China Scholarships Council (CSC 201906010314). 


\section{Statement of Ethics}

An ethics statement is not applicable because this study is based exclusively on published literature.

\section{Conflict of Interest Statement}

The authors have no conflicts of interests to declare.

\section{Funding Sources}

This study is funded by the NIHR [Programme Grants for Applied Research (NIHR202339)]. The views expressed are those of the author(s) and not necessarily those of the NIHR or the Department of Health and Social Care.

\section{Author Contributions}

Conceptualization was conceived by Li Zhang, Matthew D.L. O'Connell, and Yanzhong Wang. Formal analysis was performed by Li Zhang and Xianqi Li. Supervision was performed by Matthew D.L. O'Connell and Yanzhong Wang. Writing-original draft was performed by Li Zhang. Writing-review and editing were performed by Charles D.A. Wolfe, Matthew D.L. O'Connell, and Yanzhong Wang.

\section{Data Availability Statement}

All data generated or analyzed during this study are included in this article or its supplementary material files. Further inquiries can be directed to the corresponding author.

\section{References}

1 Katan M, Luft A. Global burden of stroke. Semin Neurol. 2018;38(2):208-11.

2 World Health Organization [Internet]. Projections of mortality and causes of death, 2016 and 2060 [cited 2021 April 16]. Available from: https://www.who.int/healthinfo/global_burden_disease/projections/en/.

3 Mohan KM, Wolfe CD, Rudd AG, Heuschmann PU, Kolominsky-Rabas PL, Grieve AP. Risk and cumulative risk of stroke recurrence: a systematic review and meta-analysis. Stroke. 2011 Jan;42(5):1489-94.

4 Guzik A, Bushnell C. Stroke epidemiology and risk factor management. Continuum. 2017 Feb;23(1):15-39.

5 Cho NH, Whiting D, Forouhi N. IDF Diabetes Atlas. ed; 2015 [cited 2021 April 16]. Available from: www.diabetesatlas.org.

6 Cavender MA, Scirica BM, Raz I, Steg PG, McGuire DK, Leiter LA, et al. Cardiovascular outcomes of patients in SAVOR-TIMI 53 by baseline hemoglobin A1c. Am J Med. 2016 Mar;129(3):340-8.

7 O'Donnell MJ, Chin SL, Rangarajan S, Xavier $\mathrm{D}$, Liu L, Zhang H, et al. Global and regional effects of potentially modifiable risk factors associated with acute stroke in 32 countries (INTERSTROKE): a case-control study. Lancet. 2016 Aug;388(10046):761-75.

$8 \mathrm{Hu}$ G, Jousilahti P, Sarti C, Antikainen R, Tuomilehto J. The effect of diabetes and stroke at baseline and during follow-up on stroke mortality. Diabetologia. 2006 Aug;49(10): 2309-16.

9 Emerging Risk Factors Collaboration; Sarwar N, Gao P, Seshasai SR, Gobin R, Kaptoge S, et al. Diabetes mellitus, fasting blood glucose concentration, and risk of vascular disease: a collaborative meta-analysis of 102 prospective studies. Lancet. 2010 Jun;375(9733): 2215-22.
10 Lau LH, Lew J, Borschmann K, Thijs V, Ekinci EI. Prevalence of diabetes and its effects on stroke outcomes: a meta-analysis and literature review. J Diabetes Investig. 2019 May; 10(3):780-92.

11 Shou J, Zhou L, Zhu S, Zhang X. Diabetes is an independent risk factor for stroke recurrence in stroke patients: a meta-analysis. J Stroke Cerebrovasc Dis. 2015 Sep;24(9): 1961-8.

12 Sibolt G, Curtze S, Melkas S, Pohjasvaara T, Kaste M, Karhunen PJ, et al. Post-stroke depression and depression-executive dysfunction syndrome are associated with recurrence of ischaemic stroke. Cerebrovasc Dis. 2013 Oct;36(5-6):336-43.

13 Adams HP, Jr, Bendixen BH, Kappelle LJ, Biller J, Love BB, Gordon DL, et al. Classification of subtype of acute ischemic stroke. Definitions for use in a multicenter clinical trial. TOAST. Trial of Org 10172 in acute stroke treatment. Stroke. 1993 Jan;24(1):35-41.

14 Bamford J, Sandercock P, Dennis M, Burn J, Warlow C. Classification and natural history of clinically identifiable subtypes of cerebral infarction. Lancet. 1991 Jun;337(8756):15216.

15 Wells GA, Shea B, O'Connell D, Peterson J, Welch V, Losos M, et al. The Newcastle Ottawa Scale (NOS) for assessing the quality of nonrandomised studies in meta-analyses; [cited 2021 April 16]. Available from: http:// www.ohri.ca/programs/clinical_epidemiology/oxford.asp.

16 Higgins JP, Altman DG, Gøtzsche PC, Jüni P, Moher D, Oxman AD, et al. The cochrane collaboration's tool for assessing risk of bias in randomised trials. Bmj. 2011 Oct;343:d592837.

17 Higgins JP, Thompson SG. Quantifying heterogeneity in a meta-analysis. Stat Med. 2002 Jun;21(11):1539-58.
18 Begg CB, Mazumdar M. Operating characteristics of a rank correlation test for publication bias. Biometrics. 1994 Dec;50(4):1088-101.

19 Piernik-Yoder B, Ketchum N. Rehabilitation outcomes of stroke patients with and without diabetes. Arch Phys Med Rehabil. 2013 Apr 94(8):1508-12.

20 Tanaka R, Ueno Y, Miyamoto N, Yamashiro $\mathrm{K}$, Tanaka Y, Shimura H, et al. Impact of diabetes and prediabetes on the short-term prognosis in patients with acute ischemic stroke. Neurol Sci. 2013 Sep;332(1-2):45-50.

21 Lei C, Wu B, Liu M, Chen Y. Association between hemoglobin $\mathrm{A}_{1} \mathrm{C}$ levels and clinical outcome in ischemic stroke patients with or without diabetes. J Clin Neurosci. 2015 Mar; 22(3):498-503.

22 Ripley DL, Seel RT, Macciocchi SN, Schara SL, Raziano K, Ericksen JJ. The impact of diabetes mellitus on stroke acute rehabilitation outcomes. Am J Phys Med Rehabil. 2007 Sep; 86(9):754-61.

23 Lee AH, Somerford PJ, Yau KK. Risk factors for ischaemic stroke recurrence after hospitalisation. Med J Aust. 2004 Sep;181(5):244-

24 Putaala J, Haapaniemi E, Metso AJ, Metso TM, Artto V, Kaste M, et al. Recurrent ischemic events in young adults after first-ever ischemic stroke. Ann Neurol. 2010 Nov; 68(5):661-71.

25 MacDougal EL, Herman WH, Wing JJ, Morgenstern LB, Lisabeth LD. Diabetes and ischaemic stroke outcome. Diabet Med. 2018 May;35:1249-57.

26 Brown DL, Shafie-Khorassani F, Kim S, Chervin RD, Case E, Morgenstern LB, et al. Sleep-disordered breathing is associated with recurrent ischemic stroke. Stroke. 2019 Mar; 50(3):571-6. 
27 Chaudhary D, Khan A, Shahjouei S, Gupta M, Lambert C, Avula V, et al. Trends in ischemic stroke outcomes in a rural population in the United States. J Neurol Sci. 2021 Mar;422: 117339.

28 Soda T, Nakayasu H, Maeda M, Kusumi M, Kowa H, Awaki E, et al. Stroke recurrence within the first year following cerebral infarction: Tottori University Lacunar Infarction Prognosis Study (TULIPS). Acta Neurol Scand. 2004 Dec;110(6):343-9.

29 Xu G, Liu X, Wu W, Zhang R, Yin Q. Recurrence after ischemic stroke in Chinese patients: impact of uncontrolled modifiable risk factors. Cerebrovasc Dis. 2007 Nov;23(2-3): $117-20$.

30 Jing J, Pan Y, Zhao X, Zheng H, Jia Q, Li H, et al. Prognosis of ischemic stroke with newly diagnosed diabetes mellitus according to hemoglobin A1c criteria in Chinese population. Stroke. 2016 Aug;47(8):2038-44.

31 Lv Y, Fang X, Asmaro K, Liu H, Zhang X, Zhang $\mathrm{H}$, et al. Five-year prognosis after mild to moderate ischemic stroke by stroke subtype: a multi-clinic registry study. PLoS One. 2013 Nov;8(11):e75019.

32 Yokota C, Minematsu K, Ito A, Toyoda K, Nagasawa $\mathrm{H}$, Yamaguchi T. Albuminuria, but not metabolic syndrome, is a significant predictor of stroke recurrence in ischemic stroke. J Neurol Sci. 2009 Feb;277(1-2):50-3.

33 Hart RG, Pearce LA, Bakheet MF, Benavente OR, Conwit RA, McClure LA, et al. Predictors of stroke recurrence in patients with recent lacunar stroke and response to interventions according to risk status: secondary prevention of small subcortical strokes trial. J Stroke Cerebrovasc Dis. 2014 Apr;23(4):618-24

34 Park HJ, Kim KW, Won SE, Yoon S, Chae YK, Tirumani SH, et al. Definition, incidence, and challenges for assessment of hyperprogressive disease during cancer treatment with immune checkpoint inhibitors: a systematic review and meta-analysis. JAMA Netw Open. 2021 Mar;4(3):e211136.

35 Zhao M, Deng XF, Gao FL, Zhang D, Wang S, Zhang $\mathrm{Y}$, et al. Ischemic stroke in young adults with moyamoya disease: prognostic factors for stroke recurrence and functional outcome after revascularisation. World Neurosurg. $2017 \mathrm{Jul} ; 103: 161-7$.

36 Type 2 Diabetes [cited 2021 April 16]. Available from: https://www.diabetes.co.uk/type2diabetes.html.

37 Furie KL, Kasner SE, Adams RJ, Albers GW, Bush RL, Fagan SC, et al. Guidelines for the prevention of stroke in patients with stroke or transient ischemic attack: a guideline for healthcare professionals from the American heart association/american stroke association. Stroke. 2011 Jan;42(1):227-76.

38 Mitchell EA, Coates VE, Ryan AA, McCarron MO, Lyttle D, McCrum-Gardner E. Hyperglycaemia monitoring and management in stroke care: policy vs. practice. Diabet Med. 2012 Sep;29(9):1108-14.
39 Dave JA, Engel ME, Freercks R, Peter J, May $\mathrm{W}$, Badri M, et al. Abnormal glucose metabolism in non-diabetic patients presenting with an acute stroke: prospective study and systematic review. QJM. 2010 Jul;103(7):495503.

40 Vancheri F, Curcio M, Burgio A, Salvaggio S, Gruttadauria G, Lunetta MC, et al. Impaired glucose metabolism in patients with acute stroke and no previous diagnosis of diabetes mellitus. QJM. 2005 Dec;98(12):871-8.

41 Tapia-Pérez JH, Gehring S, Zilke R, Schneider T. Effect of increased glucose levels on shortterm outcome in hypertensive spontaneous intracerebral hemorrhage. Clin Neurol Neurosurg. 2014 Mar;118:37-43.

42 Kimura K, Iguchi Y, Inoue T, Shibazaki K, Matsumoto N, Kobayashi K, et al. Hyperglycemia independently increases the risk of early death in acute spontaneous intracerebral hemorrhage. J Neurol Sci. 2007 Apr;255(1-2): 90-4.

43 Saxena A, Anderson CS, Wang X, Sato S, Arima H, Chan E, et al. Prognostic significance of hyperglycemia in acute intracerebral hemorrhage: the INTERACT2 study. Stroke. 2016 Mar;47(3):682-8.

44 Wu S, Shi Y, Wang C, Jia Q, Zhang N, Zhao $\mathrm{X}$, et al. Glycated hemoglobin independently predicts stroke recurrence within one year after acute first-ever non-cardioembolic strokes onset in a Chinese cohort study. PLoS One. 2013 Nov;8(11):e80690.

45 Liu X, Wang QW, Zhu RX. Influence of miRNA Gene Polymorphism on Recurrence and Age at Onset of Ischemic Stroke in a Chinese Han Population. Neurotox Res. 2019 Dec; 37(4):781-7.

46 Yuan K, Chen JJ, Xu PF, Zhang XH, Gong $\mathrm{XQ}, \mathrm{Wu} \mathrm{M}$, et al. A Nomogram for Predicting Stroke Recurrence Among Young Adults. Stroke. 2020 April;51(6):1865-7.

47 Liu YY, Zhang M, Bao HM, Zhang ZX, Mei YQ, Yun WW, et al. The efficacy of intravenous thrombolysis in acute ischemic stroke patients with white matter hyperintensity. Brain Behav. 2018 Sep;8(12).

48 Yasaka M, Koretsune Y, Yamashita T, Oda E, Matsubayashi D, Kaburagi J, et al. Recurrent Stroke and Bleeding Events after Acute Cardioembolic Stroke-Analysis Using Japanese Healthcare Database from Acute-Care Institutions. J Stroke Cerebrovasc Dis. 2018 April; 27(4):1012-24.

49 Bergström L, Irewall AL, Söderström L, Ögren J, Laurell K, Mooe T. One-Year Incidence, Time Trends, and Predictors of Recurrent Ischemic Stroke in Sweden From 1998 to 2010: An Observational Study. Stroke. 2017 Feb;48(8):2046-51.
50 Kang K, Park TH, Kim N, Jang MU, Park SS, Park JM, et al. Recurrent Stroke, Myocardial Infarction, and Major Vascular Events during the First Year after Acute Ischemic Stroke: The Multicenter Prospective Observational Study about Recurrence and Its Determinants after Acute Ischemic Stroke I. J Stroke Cerebrovasc Dis. 2016 Jan;25(3):656-64.

51 Zhu S, McClure LA, Lau H, Romero JR, White CL, Babikian V, et al. Recurrent vascular events in lacunar stroke patients with metabolic syndrome and/or diabetes. Neurology. 2015 Aug;85(11):935-41.

52 Toni D, Di Angelantonio E, Di Mascio MT, Vinisko R, Bath PM, et al. Types of stroke recurrence in patients with ischemic stroke: a substudy from the PRoFESS trial. Int J Stroke. 2014 Oct;9(7):873-8.

53 Fang X, Li L, Zhang X, Liu H, Zhang H, Qin $\mathrm{X}$. Long-term prognosis and prognostic determinants of patients with first attack of mild and moderate ischemia at Beijing community hospitals. Neural Regen Res. 2012 Mar;7(7): 540-5.

54 Suzuki N, Sato M, Houkin K, Terayama Y, Uchiyama S, Daida H, et al. One-year atherothrombotic vascular events rates in outpatients with recent non-cardioembolic ischemic stroke: the EVEREST (Effective Vascular Event REduction after STroke) registry. J Stroke Cerebrovasc Dis. 2012 Mar;21(4):24553.

55 Shinohara Y, Gotoh F, Tohgi H, Hirai S, Terashi A, Fukuuchi Y, et al. Antiplatelet cilostazol is beneficial in diabetic and/or hypertensive ischemic stroke patients. Subgroup analysis of the cilostazol stroke prevention study. Cerebrovasc Dis. 2008 May;26(1):6370.

56 Han JH, Ho SS, Lam WW. Total cerebral blood flow estimated by color velocity imaging quantification ultrasound: a predictor for recurrent stroke? J Cereb Blood Flow Metab. 2007 Sep;27(4):850-6.

57 Appelros P, Samuelsson M, Lindell D. Lacunar infarcts: functional and cognitive outcomes at five years in relation to MRI findings. Cerebrovasc Dis. 2005 June;20(1):3440.

58 Tsivgoulis G, Spengos K, Zakopoulos N, Manios E, Xinos K, Vassilopoulos D, et al. Twenty four hour pulse pressure predicts long term recurrence in acute stroke patients. J Neurol Neurosurg Psychiatry. 2005 Jan; 76(10):1360-5.

59 Kaplan RC, Tirschwell DL, Longstreth WT, Manolio TA, Heckbert SR, Lefkowitz D, et al. Vascular events, mortality, and preventive therapy following ischemic stroke in the elderly. Neurology. 2005 June;65(6):835-42.

60 Hier DB, Foulkes MA, Swiontoniowski M, Sacco RL, Gorelick PB, Mohr JP, et al. Stroke recurrence within 2 years after ischemic infarction. Stroke. 1991 Feb;22(2):155-61. 